Strategischer Zugang zum Recht 


\title{
Strategische Prozessführung - Potentiale und Risiken transnationaler zivilgesellschaftlicher Zuflucht zum Recht
}

\author{
Vera Strobel
}

Abstract: Um gezielten Zugang zum Recht zu erreichen, breitet sich in Deutschland die Rechtspraxis der strategischen Prozessführung immer weiter aus. Hierdurch streben zivilgesellschaftliche Akteure einen strategischen gerichtlichen Zugang an, der insbesondere in Fällen mit Auslandsbezug und bezüglich völkerrechtlicher Argumente bemerkenswert ist. Dieses Phänomen lässt sich als Zuflucht zu den Gerichten mit rechtspolitischen Zielen und mithilfe eines gezielten Rückgriffs auf rechtliche Instrumentarien beschreiben. Damit stellen sich Herausforderungen für die Rolle der Judikative und darüber hinaus für die Rechtsordnung als solche. In diesem Zusammenhang eröfnen sich jedoch auch Potentiale für den Rechtsschutz und für den Rechtszugang insgesamt.

\section{Aktuelle Entwicklungen und Begriff der strategischen Prozessführung}

Strategische Prozessführung nimmt weltweit und auch in Deutschland zu. ${ }^{1}$ Als strategisch geführte Prozesse werden im Rahmen dieses Beitrags Verfahren bezeichnet, die über die Durchsetzung der subjektiven Rechte Einzelner hinausgehen und rechtsgestaltende oder gesellschaftspolitische (Folge-)Wirkungen anvisieren, wofür die Akteur:innen gezielt Kläger:innen und Foren auswählen. ${ }^{2}$ Über Einzelheiten der Begriffserfassung und Einordnung als strategische Prozessführung besteht - da es sich um ein dynamisches Phänomen der Rechtspraxis handelt - bisher keine Einigkeit in der Literatur und unter den Beteiligten. ${ }^{3}$ Als rechtspraktisches Vorgehen zielt strategische Prozessführung durch zivilgesellschaftliches Engagement

1 Helmrich, Strategic Litigation rund um die Welt, in: Graser/Helmrich (Hrsg.), Strategic Litigation. Begriff und Praxis, 2019, S. 115; so auch Hahn, Strategische Prozessführung. Ein Beitrag zur Begriffsklärung, ZfRS 39 (2019), 5 (6 - 7).

2 Vgl. Graser, Was es über Strategic Litigation zu schreiben gälte, in: Graser/Helmrich, Strategic Litigation. Begriff und Praxis, 2019, S. 9 (11).

3 Hahn (Fn. 1), 8 - 11; Müller, Begriffe, Ansprüche und deren Wirklichkeiten. Ein Systematisierungsvorschlag für sogenannte strategische Prozessführung, cause lawyering und andere Formen intentional gesellschaftsgestaltender Rechtspraxen, 
auf die gerichtliche Überprüfung bestimmter Sachverhalte und Rechtsnormen ab und wirkt damit auf die (Durch-)Setzung und Auslegung von Recht sowie auf den rechtspolitischen Diskurs und die Öffentlichkeit ein. ${ }^{4}$

Strategische Prozesse gehen dabei vermehrt über rein nationale Sachverhalte hinaus und betreffen auch transnationale und internationale Fragestellungen, auf die sich dieser Beitrag insbesondere konzentrieren wird. Besonders bemerkenswert ist hierbei, dass in strategischen Prozessen vor nationalen Gerichten vermehrt völkerrechtliche - neben menschenrechtlichen sogar humanitär-völkerrechtliche - Argumente vorgetragen werden. Im Fokus dieser strategischen Verfahren standen zuletzt beispielsweise extraterritoriale grundrechtliche Schutzpflichten bezüglich US-Drohneneinsätzen im Jemen, ${ }^{5}$ Ermittlungspflichten hinsichtlich deutscher Auslandseinsätze in Afghanistan, ${ }^{6}$ sogenannte „Klimaklagen“ im Umweltrecht, ${ }^{7}$ extraterritoriale Grundrechtsbindungen bei Eingriffen des Bundesnachrichtendienstes im Ausland ${ }^{8}$ die Unternehmensverantwortung innerhalb globaler Lieferketten ${ }^{9}$ sowie Konstellationen aus dem Asyl- und Flüchtlingsrecht. ${ }^{10}$

ZfRS 39 (2019), 33 (34 - 43); umfassend das Werk Graser/Helmrich (Hrsg.), Strategic Litigation: Begriff und Praxis, Baden-Baden, 2019.

4 Duffy, Strategic Human Rights Litigation: Understanding and Maximising Impact, 2018, S. $60-62$.

5 Seit dem 22. März 2021 anhängige Verfassungsbeschwerde vor dem BVerfG, 2 BvR 508/21; BVerwG, Urt. v. 25.11.2020 - 6 C 7.19; OVG Münster, Urt. v. 19.3.2019 - 4 A 1361/15, NJW 2019, 1898.

6 EGMR, Urt. v. 16.2.2021 - Nr. 4871/16, ECLI:CE:ECHR:2021:0216JUD000487116 (Hanan v. Germany); BVerfG, Beschl. v. 19.5.2015 - 2 BvR 987/11, NJW 2015, 3500 (Kunduz-Fall).

7 Bspw. die Verfassungsbeschwerde eingereicht am 13.01.2020 von jungen Klimaaktivist:innen unter anderem zugehörig zu Fridays for Future; die Beschwerden mehrerer Kinder vor dem UN-Ausschuss für die Rechte des Kindes, Sacchi et al. v. Argentina et al., 23.9.2019; EuGH, Urt. v. 25.3.2021 - C-565/19 P, ECLI:EU:C:2021:252 - Carvalho u.a./ Parlament und Rat (People's Climate Case).

8 Bspw. BVerfG, Urt. v. 19.5.2020 - 1 BvR 2835/17, NJW 2020, 2235 (Verfassungswidrigkeit der derzeitigen Ausland-Ausland-Fernmeldeaufklärung geregelt im BND-Gesetz).

9 Bspw. LG Dortmund, Beschl. v. 10.1.2019 - 7 O 95/15 (Schmerzensgeldklage nach Fabrikbrand in Pakistan).

10 Bspw. EGMR, Urt. v. 13.2.2020 - 8675/15, 8697/15, NVwZ 2020, 697 (N.D. and N.T. v. Spain) (sog. „heiße“ Abschiebungen). 


\section{Akteur:innen und Instrumente strategischer Prozessführung}

Als Akteur:innen in der rechtlichen Unterstützung Betroffener durch strategische Prozessführung sind besonders spezialisierte Nichtregierungsorganisationen und Anwält:innen hervorzuheben. Insbesondere eine transnationale Netzwerkbildung innerhalb und zwischen beratenden, prozessvertretenden und unterstützenden Akteur:innen ist hierbei zu verzeichnen. Als frühe strategische Zusammenschlüsse können in den USA die Litigation-Organisationen Center for Constitutional Rights (CCR) und American Civil Liberties Union (ACLU), beide mit Sitz in New York, aufgeführt werden. ${ }^{11}$ In Deutschland haben sich im Jahr 2007 das European Center for Constitutional and Human Rights e.V. (ECCHR) ${ }^{12}$, im Jahr 2015 die Gesellschaft für Freiheitsrechte e.V. (GFF) ${ }^{13}$ sowie im Jahr 2016 JUMEN e.V. - Juristische Menschenrechtsarbeit in Deutschland ${ }^{14}$ gegründet und sich zu den bekanntesten Akteur:innen der strategischen Prozessführung im grund- und menschenrechtlichen Bereich hierzulande entwickelt. Diese stellen sog. Klageorganisationen dar, die sich insbesondere auf strategisch geführte Verfahren spezialisiert haben und - laut eigenem Selbstverständnis - durch gezieltes, juristisches Engagement zur nachhaltigen und effektiven Verwirklichung von individuellen Rechten beitragen wollen. ${ }^{15}$

In der deutschen Rechtskultur sind diese noch nicht derart etabliert wie in anderen Rechtssystemen, beispielsweise den USA. ${ }^{16} \mathrm{Zu}$ den Errungenschaften des gesellschaftlichen und rechtlichen Fortschritts - erreicht, inter alia, durch Urteile im Rahmen von strategisch geführten Präzedenzfällen gehörten in den USA insbesondere Fälle aus der Anti-Rassismus-Bewegung

11 CCR, About Us, https://ccrjustice.org/home/who-we-are/mission-and-vision (letzter Aufruf am 2.4.2021); ACLU, About the ACLU, https:/www.aclu.org/abo ut-aclu (letzter Aufruf am 2.4.2021).

12 ECCHR, Wer Wir Sind, https://www.ecchr.eu/ueber-uns/ (letzter Aufruf am 2.4.2021).

13 Burghardt/Thönnes, Die Gesellschaft für Freiheitsrechte, in: Graser/Helmrich (Hrsg.), Strategic Litigation, 2019, S. 65.

14 Kessler/Borkamp, JUMEN e.V. - mit juristischen Mitteln für die praktische Umsetzung der Grund- und Menschenrechte in Deutschland, in: Graser/Helmrich (Hrsg.), Strategic Litigation, 2019, S. 73.

15 Vgl. Burghardt/Thönnes (Fn. 13), S. 66 - 69; Keller/Theurer, Menschenrechte mit rechtlichen Mitteln durchsetzen: Die Arbeit des ECCHR, in Graser/Helmrich, Strategic Litigation, 2019, S. 53 (53-54).

16 Graser (Fn. 2), S. 11; Kaleck, Mit Recht gegen die Macht, in: Graser/Helmrich, Strategic Litigation, 2019, S. 21. 
sowie der Frauenrechtsbewegung. ${ }^{17}$ Wichtige Verfahren fanden in den letzten Jahrzehnten jedoch neben dem globalen Norden ebenso schon viel früher im Rahmen von public interest litigation im globalen Süden statt, insbesondere in Indien und Pakistan. ${ }^{18}$ Mit der in Deutschland in den letzten Jahrzehnten gewachsenen professionellen juristischen Unterstützung von Betroffenengruppen treten - im Gegensatz zu klassischen Prozessbeteiligten und anders als oftmals überwiegend politisch und nur peripher gerichtlich agierende NGOs - neue Akteur:innen mit öffentlichkeitswirksamen rechtspolitischen Forderungen auf die Bühne der Gerichte.

Strategische Akteur:innen treffen im Vorfeld von Prozessführung eine bestimmte Fallauswahl bezüglich taktisch eruierter Sachverhaltskonstellationen und Kläger:innen, die sie als besonders erfolgsversprechend oder als beispielhaft für die geltend gemachten Rechtsprobleme ansehen. ${ }^{19}$ Dies ist vor dem Hintergrund des überwiegend unzulässigen direkten Vorgehens von nicht subjektiv Betroffenen im Rahmen von Klage- und Beschwerdebefugnis zu sehen. ${ }^{20}$ Die Akteur:innen nehmen ebenso eine Auswahl von bestimmten Foren vor, insbesondere in Bezug auf das Gericht und den Rechtsweg. Besonders die Rechtswegerschöpfung spielt eine wichtige Rolle für den Weg zu den höchsten Gerichten, um eine Entscheidung von besonderer Tragweite zu erreichen. Weiterhin entscheiden sich Prozessbeteiligte zwischen nationalen, ausländischen und internationalen Rechtswegen sowie behördlichen, gerichtlichen und quasi-gerichtlichen Verfahrensmöglichkeiten vor dem Hintergrund ihrer jeweiligen prozessualen Voraussetzungen sowie möglicher Folgen hierdurch. ${ }^{21}$ Konkrete Rechtszugangsfragen gehören damit zu den strategischen Erwägungen im Vorfeld von Prozessführung und gestalten den Rahmen strategischer Prozessführung maßgeblich aus.

Instrumente strategischer Prozessführung im engeren Sinne stellen Anträge und Widerspruchsverfahren bei Behörden und Institutionen sowie gerichtliche Klagen und Beschwerden dar. In einem weiten Sinne lassen

17 Brown v Board of Education of Topeka, 347 U.S. 483 (1954); Weiss, The Essence of Strategic Litigation, in: Graser/Helmrich, Strategic Litigation, 2019, S. 27.

18 Khan, Genesis and Evolution of Public Interest Litigation in the Supreme Court of Pakistan: Toward a Dynamic Theory of Judicialization, Temp. Int'l \& Comp L.J. 28 (2014), S. 285 (285-291).

19 Duffy, Strategic Human Rights Litigation (Fn. 4), S. 250 - 254; Lange, Auf der Suche nach dem idealen Beschwerdeführer, ZRP 50 (2017), 18 (18 - 20); Burghardt/ Thönnes (Fn. 13), S. $69-70$.

20 S. dazu ausführlich Masing, Die Mobilisierung des Bürgers für die Durchsetzung des Rechts, 1997, S. $89-111$.

21 Duffy, Strategic Human Rights Litigation (Fn. 4), S. $254-256$. 
sich auch Strafanzeigen und das Auftreten für Nebenkläger:innen, Prozessbeobachtung und -berichte, ${ }^{22}$ sowie die Abgabe von Expert:innenmeinungen und Stellungnahmen, insbesondere vor Gericht und im Gesetzgebungsprozess unter die Tätigkeiten strategischer Akteur:innen fassen. ${ }^{23}$ Dies alles stellt zunächst eine gesetzlich vorgesehene Teilnahme am gerichtlichen und parlamentarischen Verfahren dar. Neu sind jedoch die auf Prozessführung spezialisierten Netzwerke und Zusammenschlüsse mit Herangehensweisen bezüglich bestimmter Grund- und Menschenrechte mit gemeinwohlorientierten rechtspolitischen Motiven. Damit agieren diese als Klagekollektive ${ }^{24}$ und unterscheiden sich somit von herkömmlichen NGOs sowie wirtschaftlicher Lobbyarbeit oder Interessensvertretung. ${ }^{25}$ Insbesondere kann man hierdurch ein Verlassen der politischen Sphäre ${ }^{26}$ und ein Betreten der judiziellen Sphäre mit rechtspolitischen Gestaltungsansprüchen verzeichnen.

\section{Rahmenbedingungen und Voraussetzungen für strategische Verfahren}

Wichtige Rahmenbedingungen, in denen strategische Prozessführung operiert, sind in prozessrechtlicher Hinsicht - wie für Rechtsmobilisierung im Allgemeinen - Vorschriften über Zuständigkeiten und Zulässigkeitsanforderungen sowie die Beweislast und Verfahrenskosten. ${ }^{27}$ Weiterhin ist die Bereitschaft der klagebefugten Personen notwendig, Teil eines ge-

22 S. Tätigkeiten des ECCHR selbstbezeichnet als „juristische Intervention“ insbesondere im Bereich des Völkerstrafrechts wie die Anzeige und Prozessbegleitung von Straftaten gegen das Völkerrecht in Syrien vor dem OLG Koblenz, Strafverfahren - 1 StE 9/19 und hierbei die Verurteilung eines mutmaßlichen Mitarbeiters des syrischen Geheimdienstes wegen Beihilfe zu einem Verbrechen gegen die Menschlichkeit, OLG Koblenz, Urt. v. 24.2.2021 - 1 StE 3/21; Keller/Theurer (Fn. 15), S. $53-56$.

23 Burghardt/Thönnes (Fn. 13), S. $70-71$.

24 Hahn (Fn. 1), $19-21$.

25 So auch Müller, Begriffe, Ansprüche und deren Wirklichkeiten. Ein Systematisierungsvorschlag für sogenannte strategische Prozessführung, cause lawyering und andere Formen intentional gesellschaftsgestaltender Rechtspraxen, ZfRS 39 (2019), 33 (41).

26 Fuchs, Rechtsmobilisierung. Rechte kennen, Rechte benutzen und Recht bekommen, in: Boulanger/Rosenstock/Singelnstein (Hrsg.), Interdisziplinäre Rechtsforschung, 2017, S. 243 (252).

27 Baer, Rechtssoziologie, 2020, S. 235 - 236.; hierzu auch Graser, Zugang zum Recht: Kein Thema für die deutsche (Sozial-)Rechtswissenschaft? ZIAS (2020), 13 $(25-26)$. 
richtlichen Verfahrens zu werden. In diesem Zusammenhang ist auf das bemerkenswerte Konzept der public interest litigation, das Ähnlichkeiten zur strategic litigation hierzulande aufweist, hinzuweisen. Dieses ist besonders in Indien und Pakistan seit Jahrzehnten verbreitet und ermöglicht prozessual, dass Unbeteiligte die Menschenrechte, insbesondere von vulnerablen und marginalisierten Personengruppen, einklagen können und so erweiterte Rechtszugänge geschaffen werden. ${ }^{28}$ Generell gilt es in transnationalen Fällen Sprachbarrieren und Unkenntnis über die eigenen Rechte sowie über andere Rechtssysteme zu überwinden. ${ }^{29}$ Denn Zugang zu rechtlichem sowie tatsächlichem Wissen ist ein essentieller Faktor, um den strategischen Zugang zu Gerichten sicherzustellen. Problematisch sind deshalb auch heimliche Eingriffe in Grund- und Menschenrechte. ${ }^{30}$ Deshalb können Berichte durch sogenannte Whistleblower entscheidend für die Kenntnis von etwaigen Rechtsverletzungen sowie für die Beweisführung sein. ${ }^{31}$

Insgesamt stellen damit Fragen des Zugangs zum Recht auch immer Rahmenbedingungen dar, in denen strategische Prozessführung operiert. Gerade im Bereich der extraterritorialen Anwendbarkeit von Grund- und Menschenrechten ist vieles noch ungeklärt, sodass in diesen Fällen häufig von strategischer Prozessführung zur Erreichung eines solchen Zugangs zum Recht Gebrauch gemacht wird. Dies zeigt sich beispielsweise in dem aktuellen Urteil des Europäischen Gerichtshofs für Menschenrechte im Fall Hanan gegen Deutschland, in welchem der Gerichtshof vor materiell-rechtlicher Würdigung der vorgebrachten Menschenrechtsverletzungen bei Ermittlungen zu Auslandseinsätzen in Afghanistan zunächst ausführlich seine eigene Zuständigkeit prüft. Damit wird die extraterritoriale Reichweite der EMRK thematisiert, wobei der Gerichtshof nur aufgrund

28 Bhuwania, Courting the People. Public Interest Litigation in Post-Emergency India, 2016, S. 16 ff.; Khan (Fn. 18), 285 - 291.

29 Ausführlich hierzu Ancheita/Terwindt, Auf dem Weg zu einer funktionierenden transnationalen Zusammenarbeit auf Augenhöhe, Forschungsjournal Soziale Bewegung 28 (2015), 56 (58-61).

30 Heupel, Umkämpfte Verantwortungszuschreibung: Extraterritoriale Menschenrechtsverletzungen der USA in der Terrorismusbekämpfung, Politik und Verantwortung 52 (2017), 244 (248 - 250).

31 So beispielsweise die Enthüllungen von Edward Snowden für die Vorbringen der Beschwerdeführer im BND-Verfahren sowie im Ramstein-Fall zu Drohneneinsätzen, s. ECCHR, Pressemitteilung, Edward Snowden zum Urteil gegen Internetüberwachung durch den BND, https://www.ecchr.eu/pressemitteilung/edward-s nowden-zum-urteil-gegen-internetueberwachung-durch-den-bnd/ (letzter Aufruf am 2.4.2021). 
besonderer Umstände zur Bejahung seiner Zuständigkeit gelangt und mithin Rechtszugang gewährt. ${ }^{32}$ Besonders auch im Umweltrecht stellen sich bei sogenannten Klimaklagen (transnationale) Rechtszugangshürden und oftmals wird strategisch über Grund- und Menschenrechte Zugang zu Gerichten angestrebt. ${ }^{33}$

Weitere Faktoren im Sinne von politischen Rahmenbedingungen wie das Vorhandensein und die effektive Verwirklichung von Menschenrechten sowie demokratische und rechtsstaatliche Strukturen, insbesondere eine unabhängige, effektive Gerichtsbarkeit, spielen ebenso eine wichtige Rolle, um überhaupt auf Gerichte für strategische Verfahren zurückgreifen zu können. ${ }^{34}$ Gesellschaftliche und sozio-ökonomische Faktoren sowie weitere faktische Gegebenheiten sind ebenso entscheidend..$^{35}$

\section{Fallbeispiel: Das Ramstein-Verfahren vor dem Bundesverwaltungsgericht}

Im Folgenden soll als Beispiel für strategische transnationale Prozessführung das Ramstein-Verfahren, welches US-Drohneneinsätze im Jemen und grundrechtliche Schutzpflichten zum Gegenstand hat, näher betrachtet werden. Die hierzu ergangene Entscheidung des Bundesverwaltungsgerichts wird anhand ihrer prozessualen und materiell-rechtlichen Aspekte analysiert, um aufzuzeigen wie ein solcher Fall rechtsdogmatisch aufgebaut sein kann und welche strategischen Implikationen sich hieraus ergeben. ${ }^{36}$

32 EGMR, Urt. v. 16.02.2021 - Nr. 4871/16, (Hanan v. Germany), 102 - 153; hierzu Mehta, Tailoring the Jurisdiction of the ECHR: The ECtHR's Grand Chamber Decision in Hanan v. Germany, Verfassungsblog, 18.2.2021, https:/verfassung sblog.de/tailoring-the-jurisdiction-of-the-echr/ (letzter Aufruf am 2.4.2021).

33 S. bspw. People's Climate Case: EuGH, Urt. v. 25.3.2021 - C-565/19 P, ECLI:EU:C:2021:252 - Carvalho u.a./ Parlament und Rat; hierzu Klinger, Time for an Update of the EU Charter of Fundamental Rights, Völkerrechtsblog, 14.04.2021, https://voelkerrechtsblog.org/time-for-an-update-of-the-eu-charter -of-fundamental-rights/.

34 Fuchs, Rechtsmobilisierung (Fn. 26), S. $243-244$.

35 Vgl. generell hierzu Graser, Zugang zum Recht (Fn. 27), 25 - 26.

36 Ausführlich hierzu Strobel, Kein extraterritorialer Schutzanspruch - Das BVerwG zu US-Drohneneinsätzen über Ramstein, JuWiss Blog, 9.12.2020,

https://www.juwiss.de/137-2020/ (letzter Aufruf am 2.4.2021). 


\section{Verfahrenshintergrund und bisherige Gerichtsentscheidungen}

Angestoßen wurde das Verfahren vor dem Verwaltungsgericht Köln durch die Leistungsklage und hilfsweise Feststellungsklage dreier jemenitischer Staatsangehöriger, deren Angehörige als unbeteiligte Zivilisten im Jahr 2012 im Jemen durch einen US-amerikanischen Drohnenangriff getötet wurden. ${ }^{37}$ Für diese Einsätze werden die Daten von und zu bewaffneten Drohnen im Jemen über deutsches Staatsgebiet durch die Satelliten-RelaisStation auf der US Air Base Ramstein in Rheinland-Pfalz übermittelt. Die Kläger beriefen sich vor Gericht auf die Schutzpflicht hinsichtlich ihres Rechts auf Leben gemäß Art. 2 Abs. 2 S. 1 GG und verlangten, die Bundesrepublik Deutschland zu verurteilen, auf eine völkerrechtskonforme Nutzung der Air Base Ramstein durch die USA für Drohneneinsätze im Jemen mithilfe geeigneter Maßnahmen hinzuwirken oder hilfsweise die Rechtswidrigkeit des Unterlassens festzustellen. ${ }^{38}$

Die jemenitischen Kläger wurden in den Gerichtsverfahren in Deutschland vom ECCHR unterstützt. ${ }^{39}$ Dieses ist als international agierende Nichtregierungsorganisation in dem Verfahren im Rahmen ihrer Strategie der juristischen Intervention zur Bewirkung einer Durchsetzung von Menschenrechten und des Völkerrechts tätig. ${ }^{40}$ Wesentlicher Bestandteil dieser Vorgehensweise ist der gezielte Rückgriff auf rechtliche Instrumente mittels spezifischer Einzelfälle, die ihrer Einschätzung nach generelle Verstöße gegen Menschenrechte oder das Völkerrecht offenlegen. ${ }^{41}$ Dabei soll nicht nur die Durchsetzung von Rechten und Ansprüchen Betroffener erreicht, sondern darüber hinaus auch eine bestimmte rechtspolitische Zielsetzung verfolgt werden. Letztere liegt hier in einem Vorgehen gegen die Betei-

37 VG Köln, Urt. v. 27.5.2015 - 3 K 5625/14, Rn. 2 - 4; Bin Ali Jaber, Germany Could Have Delivered Justice for Civilian Drone Strike Victims. It Failed, Foreign Policy, 8.12.2020, https://foreignpolicy.com/2020/12/08/germany-could-have-delivered -justice-for-civilian-drone-strike-victims-it-failed/ (letzter Aufruf am 2.4.2021); ECCHR, Fallbeschreibung, https://www.ecchr.eu/fileadmin/Fallbeschreibungen/ Fallbeschreibung_Kurzportraits_Drohnen_Jemen_Oktober2020.pdf (letzter Aufruf am 2.4.2021).

38 VG Köln, Urt. v. 27.5.2015 - 3 K 5625/14, Rn. 10 - 13.

39 ECCHR, Ramstein vor Gericht: Deutschlands Rolle bei US-Drohnenangriffen im Jemen, https:/www.ecchr.eu/fall/wichtiges-urteil-deutschland-muss-us-drohneins aetze-via-ramstein-pruefen/ (letzter Aufruf am 2.4.2021).

40 ECCHR, Wie wir arbeiten, Mit juristischen Mitteln Menschenrechte verteidigen, https://www.ecchr.eu/ecchr/mit-juristischen-mitteln-menschenrechte-verteidigen/ (letzter Aufruf am 2.4.2021).

41 Keller/Theurer (Fn. 15), S. 54 - 57. 
ligung Deutschlands an der US-amerikanischen Terrorismusbekämpfung mittels gezielter Tötungen. ${ }^{42}$ Insgesamt ist das Verfahren folglich als strategische Prozessführung einzustufen.

Dem Klägerbegehren wurde jedoch zunächst nicht entsprochen. Das Verwaltungsgericht Köln wies die Klage ab und stufte die Schutzpflicht selbst wenn man vorliegend eine solche als bestehend annehmen würde - aufgrund des weiten Entscheidungsspielraums der Exekutive als nicht verletzt ein. ${ }^{43}$ Das Oberverwaltungsgericht Münster gab der Berufung der Kläger hierauf teilweise statt. ${ }^{44}$ Es verurteilte die Bundesrepublik dazu, sich durch geeignete Maßnahmen zu vergewissern, dass eine Nutzung der Air Base für bewaffnete Drohneneinsätze durch die USA im Jemen im Einklang mit dem Völkerrecht stattfindet, und erforderlichenfalls hierauf hinzuwirken. ${ }^{45}$

\section{Die Entscheidung des Bundesverwaltungsgerichts}

Dieser Entscheidung des Berufungsgerichts folgte das Bundesverwaltungsgericht jedoch nicht und stellte auf die Revision der Bundesrepublik, vertreten durch das Bundesministerium der Verteidigung, das klageabweisende erste Urteil wieder her. ${ }^{46}$ Dabei wies es zunächst die Klage eines der Kläger mangels Klagebefugnis als unzulässig zurück, da er durch einen

42 ECCHR, Ramstein vor Gericht: Deutschlands Rolle bei US-Drohnenangriffen im Jemen, Q\&A zum rechtlichen Hintergrund, https://www.ecchr.eu/fileadmin/Q As/QA_Drohnen_Jemen_Nov2020.pdf (letzter Aufruf am 2.4.2021); Bin Ali $\bar{J}$ aber, Germany Could Have Delivered Justice for Civilian Drone Strike Victims. It Failed, Foreign Policy, 8.12.2020, https:/foreignpolicy.com/2020/12/08/germa ny-could-have-delivered-justice-for-civilian-drone-strike-victims-it-failed/ (letzter Aufruf am 2.4.2021).

43 VG Köln, Urt. v. 27.5.2015 - 3 K 5625/14, Rn. 85 - 99, ausführlich hierzu Starski, So fern und doch so nah: Drohneneinsätze im Jemen im Visier der Grundrechte, Verfassungsblog, 16.6.2015, https://verfassungsblog.de/so-fern-und-doch-so-nah-dr ohneneinsaetze-im-jemen-im-visier-der-grundrech-te/ (letzter Aufruf am 2.4.2021).

44 OVG Münster, Urt. v. 19.3.2019 - 4 A 1361/15, NJW 2019, 1898.

$45 \mathrm{Zu}$ dieser Thematik Bothe, Wegschauen verletzt das Recht auf Leben: Zum Drohnenurteil des OVG Münster, Verfassungsblog, 21.3.2019, https://verfassungsblog. de/wegschauen-verletzt-das-recht-auf-leben-zum-drohnenurteil-des-ovg-muenster/ (letzter Aufruf am 2.4.2021); sowie ausführlich Aust, US-Drohneneinsätze und die grundrechtliche Schutzpflicht für das Recht auf Leben, JZ 2020, 303 (306, 309).

46 BVerwG, Urt. v. 25.11.2020 - 6 C 7.19, ausführlich hierzu Strobel, Kein Schutzanspruch gegen Drohnenangriffe? Das Urteil des BVerwG zu US-Drohneneinsätzen im Jemen mittels Ramstein, PRIF Blog, 11.12.2020, https://blog.prif.org/2020/12/ 
Aufenthaltswechsel ins Ausland für seine eigene Sicherheit gesorgt habe, ${ }^{47}$ und verwehrt damit den Zugang zu Schutzansprüchen, wenn man sich einer Lebensgefahr durch Drohneneinsätze entzieht.

In der Begründetheit der Klage der weiteren Kläger stellt das Gericht die grundsätzlich bestehende Möglichkeit von extraterritorialen Schutzpflichten klar. ${ }^{48}$ Es verlangt dafür jedoch einen qualifizierten Bezug zum deutschen Staatsgebiet, wofür die Air Base Ramstein als vorgeblich allein technisch notwendiges Bindeglied aufgrund mangelnder Entscheidungselemente auf deutschem Territorium nicht ausreichend sei. ${ }^{49}$ Die Kläger hatten hingegen inter alia geltend gemacht, dass trotz des Auslandsbezugs keine derart strengen Anforderungen an den elementaren Lebensschutz gestellt werden dürften. Ebenso argumentiert das Gericht, dass für das Entstehen einer grundrechtlichen Schutzpflicht nicht bereits lediglich die Möglichkeit einer völkerrechtswidrigen Grundrechtsbeeinträchtigung ausreiche. ${ }^{50}$ Denn die Schutzpflicht werde erst ausgelöst, wenn basierend auf vergangenen Verstößen gegen das Völkerrecht nach ihrer Zahl und Umstände abzusehen sei, dass es zukünftig zu weiteren Beeinträchtigungen komme. ${ }^{51}$ Darüber hinaus sei bei der völkerrechtlichen Bewertung die Bandbreite der vertretbaren Rechtsauffassungen aufgrund der strukturellen Besonderheiten des Völkerrechts zu berücksichtigen. ${ }^{52}$ Die Kläger argumentierten hingegen, dass gemäß Art. 20 Abs. 3, 25, 59 Abs. 2 GG eine Rechtsbindung der Exekutive auch an das Völkerrecht bestehe, die Bundesregierung die Drohneneinsatzpraxis der USA an einer eigenen völkerrechtlichen Rechtsauffassung messen sowie ihrer internationalen Verpflichtung, auf die Einhaltung des humanitären Völkerrechts durch die USA hinzuwirken, nachkommen müsse. ${ }^{53}$

Obwohl das Gericht nicht abschließend feststellen konnte, ob die Drohneneinsätze mit den humanitär-völkerrechtlichen Normen im Einklang

11/kein-schutzanspruch-gegen-drohnenangriffe-das-urteil-des-bverwg-zu-us-drohn eneinsaetzen-im-jemen-mittels-ramstein/ (letzter Aufruf am 2.4.2021).

47 BVerwG, Urt. v. 25.11.2020 - 6 C 7.19, Rn. 23 - 25.

48 Ibid, Rn. $42-44$.

49 Ibid, Rn. $49 \mathrm{f}$.

50 BVerwG, Pressemitteilung Nr. 68/2020 vom 25.11.2020, hier abrufbar: https://www.bverwg.de/pm/2020/68 (letzter Aufruf am 2.4.2021).

51 BVerwG, Urt. v. 25.11.2020 - 6 C 7.19, Rn. 51 - 54.

52 Ibid, Rn. $55-59$.

53 Diese in ihren Einzelheiten umstrittene Pflicht stellt insbesondere Art. 1 der Genfer Abkommen von 1949 auf, s. IKRK Kommentar, Art. 1 Genfer Abkommen, https://ihl-databases.icrc.org/ihl/full/GCI-commentaryArt1. (letzter Aufruf am 2.4.2021); ECCHR, (Fn. 39). 
stehen, sieht es dennoch von einer Zurückverweisung an die Tatsacheninstanz ab, da es dies nicht für entscheidungserheblich hält. ${ }^{54}$ Folglich erachtet das Gericht die Zusicherung der USA gegenüber der Bundesregierung, sie halte das Völkerrecht ein, für hinreichend - trotz von den Klägern vorgebrachter gegenteiliger Nachweise. ${ }^{55}$ Im Ergebnis lässt das Bundesverwaltungsgericht damit offen, ob eine Schutzpflicht besteht. Eine solche sei jedenfalls durch hinreichendes Tätigwerden erfüllt. Hierbei gesteht das Urteil der Exekutive einen weiten Einschätzungsspielraum bezüglich der Erfüllung der Schutzpflichten sowie aufgrund des außen- und verteidigungspolitischen Bezugs des Sachverhaltes zu. ${ }^{56}$

\section{Einordnung des Urteils und Ausblick}

Ob das Bundesverwaltungsgericht mit seinem Urteil durch die gerichtliche Zurückweisung eines individuellen Schutzanspruchs zu hohe Anforderungen an das Bestehen einer Schutzpflicht in einem Sachverhalt mit Auslandsbezug aufgestellt und damit das Recht auf Leben in seiner Schutzpflichtdimension verkannt hat, hat nun das Bundesverfassungsgericht zu entscheiden. Am 22. März 2021 haben zwei der ursprünglichen Kläger Verfassungsbeschwerde erhoben. ${ }^{57}$ Weiterhin käme hiernach gegebenenfalls eine Anrufung des Europäischen Gerichtshofs für Menschenrechte in Betracht.

Insgesamt wurde durch das bisherige Verfahren in Weiterentwicklung des sogenannten BND-Urteils ${ }^{58}$ des Bundesverfassungsgerichts die generelle Möglichkeit extraterritorialer Schutzpflichten höchstgerichtlich bestätigt. Bereits im BND-Urteil, welches durch eine strategische Verfassungsbeschwerde unterstützt von der Gesellschaft für Freiheitsrechte e.V. ermöglicht wurde ${ }^{59}$ wurde eine Grundrechtsbindung der deutschen Staatsgewalt

54 BVerwG, Urt. v. 25.11.2020 - 6 C 7.19, Rn. 60 - 66.

55 Zur Argumentation der Kläger ausführlich Strobel (Fn. 46).

56 BVerwG, Urt. v. 25.11.2020 - 6 C 7.19, Rn. 67 - 81.

57 BVerfG, Az. 2 BvR 508/21; ECCHR, Pressemitteilung, Ramstein vor dem Verfassungsgericht, https:/www.ecchr.eu/pressemitteilung/ramstein-verfassungsgericht/ (letzter Aufruf am 2.4.2021).

58 BVerfG, Urt. v. 19.5.2020 - 1 BvR 2835/17, NJW 2020, 2235 (sog. BND-Urteil); zur Dogmatik dieser Entscheidung: Schiffbauer, Die Würde des Rechtsstaats ist unantastbar, JuWissBlog v. 19.5.2020, https://www.juwiss.de/75-2020/ (letzter Aufruf am 2.4.2021).

59 GFF, BND-Gesetz, https://freiheitsrechte.org/bnd-gesetz-2/ (letzter Aufruf am 2.4.2021). 
im Ausland gegenüber sogenannten Ausländer:innen jedenfalls für die geltend gemachten Abwehrrechte angenommen. ${ }^{60}$ In beiden Fällen wurde damit das Bestehen einer umfassenden Grundrechtsbindung sowie die hieraus erwachsenden Anforderungen im Bereich der Sicherheits- und AuBenpolitik mit inhärentem Auslandsbezug verdeutlicht und damit Rechtszugang in extraterritorialen Fallkonstellationen ermöglicht. Neben grundrechtlichen Dimensionen wurde im Ramstein-Fall darüber hinaus ein $\mathrm{Zu}$ gang zu gerichtlicher Überprüfung (humanitär-)völkerrechtlicher Aspekte gestärkt, insbesondere im Hinblick auf deren Berücksichtigung bei Entscheidungen der Exekutive.

\section{Auswirkungen strategischer Verfahren}

In materiell-rechtlicher Hinsicht ist es möglich mithilfe strategischer Prozesse eine bestimmte Auslegung des einfachen Rechts oder der Verfassung sowie auf der Vollzugsebene eine bestimmte Anwendung oder Durchsetzung des gesetzten Rechts zu erreichen. ${ }^{61}$ Dabei kann eine Veränderung des geltenden Rechts ermöglicht werden, indem gesetzliche Vorschriften oder Verwaltungspraxen gerichtlich als unrechtmäßig, verfassungswidrig, europarechts- oder völkerrechtswidrig beurteilt werden. ${ }^{62}$ Weiterhin können durch strategische Prozessführung individuelle sowie kollektive Gerechtigkeit und Rechtsmittel durchgesetzt und Informationszugang erlangt werden. ${ }^{63}$ Für und mit den Betroffenen können ihre Interessen gerichtlich durchgesetzt werden - beispielsweise können eine Entschädigung in Form von Schadensersatz, Schmerzensgeld oder jedenfalls eine Verhinderung gleichartigen zukünftigen Verhaltens, der Anstoß zu Ermittlungen oder eine Verantwortungszuweisung erreicht werden. Dies kann zu gerichtlicher Anerkennung und Empowerment führen. ${ }^{64}$

Ein materiell-rechtlicher oder betroffenenspezifischer Erfolg durch strategische Prozessführung ist jedoch meist ungewiss. Statt einer erhofften Entscheidung kann eine gerichtliche Niederlage zum Nachteil der Betrof-

60 BVerfG, Urt. v. 19.5.2020 - 1 BvR 2835/17, NJW 2020, 2235, Rn. 88, 104; ausführlich dazu Gärditz, Grundrechtliche Grenzen strategischer Ausland-Ausland-Telekommunikationsaufklärung: Ein Wegweiser durch die BND-Entscheidung des BVerfG, Urteil v. 9.5.2020 - 1 BvR 2835/17, JZ 75 (2020), 825 - 835.

61 Fuchs, Rechtsmobilisierung (Fn. 26), S. 253.

62 Duffy, Strategic Human Rights Litigation (Fn. 4), S. 60 - 61.

63 Keller/Theurer (Fn. 15), S. 55 - 57.

64 Duffy, Strategic Human Rights Litigation (Fn. 4), S. 51 - 52, 59. 
fenen ausfallen, mit Auswirkungen auf die Rechtsprechung weit über den Einzelfall hinaus. ${ }^{65}$ Teils wird auch bewusst kein Verfahren angestrebt, etwa weil ablehnende Tendenzen an den Gerichten befürchtet werden. ${ }^{66}$ So kann es sein, dass eine Rechtsfrage zunächst ungeklärt bleibt.

Dagegen ist der politische Einfluss - auch bei einer juristischen Niederlage - in Form des Generierens von Aufmerksamkeit und politischer Diskussion und Meinungsbildung meist ein wichtiger Faktor, der durch strategische Beteiligte oft gezielt mit Öffentlichkeitsarbeit und Kampagnen prozessbegleitend befördert wird. ${ }^{67}$ Gleiches gilt für die gesellschaftlichen Auswirkungen durch das Schaffen von Bewusstsein für bestimmte Ungerechtigkeiten sowie die Mobilisierung von bestimmten gesellschaftlichen Gruppen durch strategische Prozesse. ${ }^{68}$ Weiterhin kann strategische Prozessführung ebenfalls institutionellen, kulturellen sowie historischen Einfluss ausüben. ${ }^{69}$ Im Einzelnen bedürfen die Folgen strategischer Verfahren weiterer Forschung und wären vor allem empirisch zu untersuchen. ${ }^{70}$

\section{Risiken, Kritik und Herausforderungen}

In Teilen der Literatur und Praxis wird den Akteur:innen strategischer Prozessführung entgegengehalten, dass ihre Herangehensweise Probleme in demokratietheoretischer und rechtsstaatlicher Hinsicht hervorrufe. ${ }^{71}$ Besonders werden die Risiken einer eventuellen missbräuchlichen Verwendung nicht für übergeordnete (rechts-)politische Fragestellungen vorgesehener Instrumente und Foren der Gerichte im Rahmen des Individual-

65 Lobel, Success Without Victory: Lost Legal Battle and the Long Road to Justice in America, 2004, S. 264 - 267.

$66 \mathrm{Zu}$ den allgemeinen Hintergründen derartiger Entscheidungsprozesse s. Hilson, New Social Movements: The Role of Legal Opportunity, JEPP 9 (2002), 238 252.

67 Lobel, Courts as Forums for Protest, UCLA Law Review 52 (2004), 477 (487 - 490); Cummings/Rhode, Public Interest Litigation: Insights From Theory and Practice, Fordham Urban Law Journal 36 (2009), 603 (615 - 619).

68 Baer (Fn. 27), S. 238 - 239.

69 Duffy, Strategic Human Rights Litigation (Fn. 4), S. 67 - 74.

70 Ein Beispiel hierfür ist Barber, Tackling the evaluation challenge in human rights: assessing the impact of strategic litigation organisations, The International Journal of Human Rights 16 (2012), 411 - 435.

71 Bspw. Wegener, Urgenda - Weltrettung per Gerichtsbeschluss? Klimaklagen testen die Grenzen des Rechtsschutzes, ZUR 1 (2019), 3 (10 ff). 
rechtsschutzes betont. ${ }^{72} \mathrm{Wiederum}$ andere Stimmen in der Literatur sehen strategische Prozessführung als „systemstabilisierend“ und damit als nicht weitgehend genug an. Dabei wird kritisiert, dass sie zu spät einsetze, ineffektive Foren adressiere und kritische Rechtspolitik auf den Rechtsstreit reduziere. Zudem weise sie mangelnde Ambitioniertheit auf und habe eine depolitisierende Tendenz. ${ }^{73}$

Gerichte hingegen weisen immer wieder die Argumentation zurück, dass mit einer bestimmten Entscheidung ihre durch das Demokratieprinzip und das Prinzip der Gewaltenteilung begrenzte richterliche Entscheidungskompetenz überschritten werde. ${ }^{74}$ Sie betonen zumeist, dass sie gegenüber der Legislative oder Exekutive lediglich bestimmte (Ziel-)Vorgaben im Rahmen ihrer Überprüfungskompetenz durchsetzten, diesen einen weiten Beurteilungs- und Entscheidungsspielraum beließen und damit gerade kein Übergriff in verfassungsrechtliche Kompetenzzuweisungen anderer Organe vorliege. ${ }^{75}$ Dennoch besteht durch die mediale Kommunikationsstrategie strategischer Akteur:innen erhöhter Druck auf die Gerichte. Insbesondere unterinstanzliche Gerichte sehen sich mit komplexen neuen Entscheidungsgegenständen wie beispielsweise humanitärem Völkerrecht konfrontiert. Gleichzeitig ist jedoch eine Zurückhaltung der Gerichte in bestimmten politischen Bereichen zu erkennen. ${ }^{76}$

$\mathrm{Zu}$ Recht werden die möglichen Unzulänglichkeiten der demokratisch schwach legitimierten Gerichte bezüglich komplexer Sachverhalte herausgehoben und betont, dass deren Rolle für die Entwicklung langfristiger Lösungskonzepte nicht zu überschätzen sei. Jedenfalls kann die Bewältigung weitgehender (globaler) Herausforderungen nicht allein durch Gerichte erfolgen. ${ }^{77}$ Prozessführung operiert im Rahmen und damit auch innerhalb der Grenzen und etwaiger Unzulänglichkeiten des vorhandenen Rechtssystems und kann dadurch ungewollt zur Perpetuierung bestehender Ungerechtigkeiten beitragen. ${ }^{78}$ Als lediglich symbolisch sind

72 Vgl. Ibid, 3.

73 Fischer-Lescano, Kassandras Recht, KJ 52 (2019), 419 (421 - 425).

74 Bspw. in der sog. Urgenda-Entscheidung der Gerechtshof Den Haag, Urt. v. 9.10.2018 - 200.178.245/01, ECLI:NL:RBDHA:2015:7196, Rn. $67 \mathrm{ff}$.

75 Ibid, Rn. 74; Wegener (Fn. 71), 5 - 6.

76 S. im Bereich der Außenpolitik, beispielsweise BVerwG, Urt. v. 25.11.2020 - 6 C 7.19, Rn. $56-81$.

77 Vgl. Wegener (Fn. 71), 3, 11.

78 Hierzu bspw. in Bezug auf das Migrationsrecht Buckel, "Welcome to Europe" die Grenzen des europäischen Migrationsrechts, Juridische Auseinandersetzungen um das "Staatsprojekt Europa", 2013, S. 38 - 39. 
beispielsweise Klimaklagen dennoch nicht zu bezeichnen, ${ }^{79}$ denn das verkennt die weitgehenden rechtlichen, politischen und gesellschaftlichen Auswirkungen, die derartige Gerichtsverfahren mit strategischer Herangehensweise bewirken können. Weiterhin ist den strategischen Beteiligten bewusst, dass allein durch bestimmte Klagen globale oder nationale Herausforderungen nicht gelöst werden können, sondern diese lediglich ein Instrument unter vielen darstellen, die in ihrem Zusammenwirken richtungsweisend sein können. ${ }^{80} \mathrm{Im}$ Vorfeld eines gerichtlichen Vorgehens im Rahmen strategischer Prozessführung erfolgt oftmals ein Einwirken im Rechtssetzungsprozess oder eine Beteiligung an der politischen Meinungsbildung zu bestehenden gesetzlichen Regelungen beziehungsweise einem Verhalten der Exekutive mithilfe zivilgesellschaftlicher Kampagnen unter Einbindung verschiedener Akteur:innen. ${ }^{81}$

Der Rückgriff auf die Judikative ist gerade in einem demokratischen Rechtsstaat vorgesehen und auch nicht bei vermeintlich (rechts-)politischen Sachverhalten verschlossen. ${ }^{82}$ Denn Recht ist zumeist gerade das Ergebnis eines politischen Prozesses. Zudem gibt es im deutschen Rechtssystem gerade keinen Ausschluss bestimmter Rechtsfragen ex ante - auch nicht rechtspolitisch umstrittener. Dass es zu einer Missachtung von Gerichtsentscheidungen - wie beispielsweise im Zusammenhang mit gerichtlich vorgegebenen Zielen des Klimaschutzes - kommen und damit eine Delegitimierung oder Destabilisierung der Judikative bewirkt werden könnte, ${ }^{83}$ führt nicht zu einer Verantwortlichkeit der prozessführenden Beteiligten hierfür, sondern ist vielmehr der Verantwortung der missachtenden Gewalt zuzuschreiben, die mit derartigem Verhalten die Funktionsweise ihres eigenen Rechtssystems gefährdet.

Weitere Risiken stellen sich durch strategische Prozesse auf internationaler Ebene wie beispielsweise Herausforderungen für die staatliche Souveränität, die gerade durch internationale Gerichtsentscheidungen entstehen und dazu führen können, dass Staaten durch vermeintlich in ihre innerstaatlichen Angelegenheiten übergreifende Entscheidungen bestimmte internationale Abkommen wie menschenrechtliche Verträge verlassen

79 Vgl. Wegener (Fn. 71), 3.

$80 \mathrm{Kahl} /$ Daebel, Climate Change Litigation in Germany An Overview of Politics, Legislation and Especially Jurisdiction regarding Climate Protection and Climate Damages, European Energy and Environmental Law Review 28 (2019), 67.

81 Keller/Theurer (Fn. 15), S. 54 - 56; Burghardt/Thönnes (Fn. 13), S. 66 - 69.

82 So auch Graser, Vermeintliche Fesseln der Demokratie: Warum die Klimaklagen ein vielversprechender Weg sind, ZUR 5 (2019), 271 (274 - 278).

83 Vgl. Wegener (Fn. 71), 3, $12 \mathrm{f}$. 
wollen. ${ }^{84}$ Zudem ist bei strategischen Prozessen zu berücksichtigen, dass Betroffenen eine bestimmte Rolle und deren Rechtsposition eine gewisse Instrumentalisierung für größere rechtspolitische Ziele zukommt, ${ }^{85}$ wodurch es zu Kollisionen zwischen individuellen und kollektiven Interessen kommen kann. Auch ist besonders bei transnationalen Verfahren im globalen Norden mit Betroffenen im globalen Süden die Rolle strategischer Akteur:innen anfällig für die Perpetuierung bestehender Ungleichheiten und Machtverhältnisse. ${ }^{86}$

\section{Potentiale und Rechtsmobilisierung}

Strategische Verfahren verändern auch die Art und Weise demokratischer Partizipation der Zivilgesellschaft. ${ }^{87}$ Auf strategische Prozessführung kann und wird zumeist von Teilen der Zivilgesellschaft mit politischer Einstellung abseits der Regierungsmehrheit für marginalisierte oder vulnerable Betroffene oder andere Minderheiten zurückgegriffen, ${ }^{88}$ wenn auf legislativer oder exekutiver Ebene keine entsprechende Veränderung bewirkt werden konnte. ${ }^{89}$ Dies rührt aus einer bestimmten rechtspolitischen, ideellen Motivation her. So will etwa die Gesellschaft für Freiheitsrechte

84 Diese Problematik erläutert abstrakt anhand der dynamischen Auslegung des EGMR van der Berg, Folter, unmenschliche und erniedrigende Behandlung in der Rechtsprechung des EGMR und die strafprozessualen Konsequenzen, 2019, S. $70 \mathrm{ff}$.

85 Lange (Fn. 19), S. 19.

86 Ausführlich hierzu Ancheita/Terwindt, Auf dem Weg zu einer funktionierenden transnationalen Zusammenarbeit auf Augenhöhe, Forschungsjournal Soziale Bewegung 28 (2015), 56 - 64; Theurer/Kaleck, Dekoloniale Rechtskritik und Rechtspraxis, 2020.

87 Hierzu ausführlich Fuchs, Strategische Prozessführung als Partizipationskanal, in: de Nève/Olteanu (Hrsg.): Politische Partizipation jenseits der Konventionen, 2013, S. $51-74$.

88 S. bspw. die Unterstützung der GFF von Verfahren zur rechtlichen Anerkennung der Elternschaft bei nicht-heterosexuellen Ehepaaren, https://freiheitsrechte.org/el ternschaft/ (letzter Aufruf am 2.4.2021) und die hierbei erreichten gerichtlichen Vorlagen an das BVerfG wegen Zweifel an der Verfassungsmäßigkeit der derzeitigen einfachgesetzlichen Regelung: OLG Celle, Beschl. v. 24.3.21 - 21 UF 146/20 und KG Berlin, Beschl. v. 24.03.2021 - 3 UF 1122/20; allgemein Thierse/Badanjak, Opposition in the EU Multi-Level Polity. Legal Mobilization against the Data Retention Directive, S. 86 - 90.

89 Fuchs, Rechtsmobilisierung (Fn. 26), S. $251-253$. 
„dem Recht zu seinem Recht verhelfen“.90 Ein derartiges gezieltes Nutzen der Kontrollfunktion der Judikative für partikulare Interessen ist wiederum nur erfolgreich, ${ }^{91}$ wenn verfassungswidrige oder unrechtmäßige Verfahrensgegenstände vorliegen. Damit besteht ein geringer politischer Gestaltungsspielraum, der jedoch im Einzelfall nicht zu unterschätzen ist, da auch rechtsdogmatisch oder politisch weitreichende Urteile nicht ausgeschlossen sind.

Durch strategische Rechtsmobilisierung wird den Gerichten die Möglichkeit eingeräumt, bestimmte Praxen oder Rechtsnormen zu beurteilen, ${ }^{92}$ die andernfalls entweder überhaupt nicht, nicht mit bestimmten Argumentationslinien oder nicht in den jeweiligen Fallkonstellationen vorgebracht worden wären. Denn Rechtsschutz wird oftmals aufgrund fehlenden Erkennens der Betroffenen, dass eine etwaige Verletzung ihrer Rechte vorliegt, oder aus finanziellen und zeitlichen Kapazitäten eines jahrelangen Verfahrens mit unsicheren Erfolgsaussichten gar nicht erst in Anspruch genommen. ${ }^{93}$ Besonders bei transnationalen Verfahren wäre es Betroffenen im Ausland durch die hohen Hürden des Rechtsschutzes in Deutschland nicht ohne Weiteres möglich, ihnen zukommende Rechte und Schutzansprüche durchzusetzen. Dies liegt nicht an vermeintlichen Unzulänglichkeiten der Betroffenen, sondern ist in dem erschwerten $\mathrm{Zu}$ gang zu Recht und Rechtsschutz sowie in Herausforderungen bei der behördlichen und gerichtlichen Anerkennung von Verantwortung der Hoheitsgewalt und bei der Zuerkennung von (Grund-)Rechten in extraterritorialen Konstellationen verwurzelt.

Somit schafft strategische Prozessführung durch eine Mobilisierung des Rechts eine intensivierte Wirkmacht bestimmter Teile der Zivilgesellschaft im Rechtsstaat, indem sie Hürden des Rechtsschutzes überwindet und praktikable Zugänge zum Recht ermöglicht. Jedoch wird durch klassische strategische Prozessführung nicht für breite Teile der Bevölkerung kollektiver Rechtszugang erreicht, wie beispielsweise durch Verbandsklagerechte, Musterfeststellungsklagen oder den strategischen Einsatz von legal

90 GFF, Team, Wir verhelfen dem Recht zu seinem Recht, https://freiheitsrechte.org /team/ (letzter Aufruf am 2.4.2021).

91 Diese Funktion bezeichnen Hahn/Fromberg als „Watchdog“ in: Klagekollektive als „Watchdogs“. Zu Chancen strategischer Prozessführung für den demokratischen Rechtsstaat, ZPol, 16.11.2020, https://doi.org/10.1007/s41358-020-00241-4 (letzter Aufruf am 2.4.2021).

92 Baer (Fn. 27), S. 238 f.; vgl. Hahn (Fn. 1), 22.

93 Vgl. Fuchs, Rechtsmobilisierung (Fn. 26), S. 246 - 247. 
tech. ${ }^{94}$ Stattdessen werden indirekt für bestimmte Betroffenengruppen partikulare Rechtsbeeinträchtigungen beseitigt, ohne dass die überwiegende Anzahl an Betroffenen hieran konkret mitwirkt. Es wird gerade durch rechtspolitische Motivation eine Mobilisierung der Rechtsdurchsetzung der eigenen Rechte bestimmter Betroffenengruppen erreicht.

\section{Fazit und Ausblick}

Rechtsmobilisierung durch strategische Prozesse ist für den individuellen und kollektiven Zugang zum Recht von Bedeutung, um etwaig verfassungswidrige oder unrechtmäßige Zustände überprüfen zu lassen ${ }^{95}$ und dadurch den Vorrang der Verfassung und effektive Rechtsdurchsetzung $\mathrm{zu}$ erreichen. Durch strategic litigation ist außerdem eine Professionalisierung der Handlungsmöglichkeiten der Zivilgesellschaft eingetreten - nicht nur national, sondern auch transnational und international. Hierdurch kann einerseits gesellschaftlicher Wandel durch gerichtliche Entscheidungen verstetigt werden und Eingang in die Rechtsordnung finden sowie andererseits durch gerichtliche Entscheidungen gesellschaftlicher und politischer Wandel bewirkt werden. Dies erfolgt jedoch nicht ohne Konsequenzen für die Rechtsordnung und Herausforderungen für die Judikative, da individuelle Rechtsmittel als rechtspolitische Gestaltungsinstrumente kollektiver Netzwerke für gezielte Prozessführung ursprünglich nicht vorgesehen waren. Es bleibt spannend, wohin sich strategische Prozessführung als dynamische Rechtspraxis in Zukunft entwickeln wird. Eine weitere Verbreitung dieses Phänomens auch in weiteren Rechtsgebieten und für Rechtsfragen im Zusammenhang mit aktuellen Herausforderungen ist jedenfalls zu erwarten.

94 Rehder/van Elten, Legal Tech \& Dieselgate. Digitale Rechtsdienstleister als Akteure der strategischen Prozessführung, ZfRS 39 (2019), 64 (67 - 71).

95 Thierse, Mobilisierung des Rechts: Organisierte Interessen und Verfassungsbeschwerden vor dem Bundesverfassungsgericht, Politische Vierteljahresschrift 61 (2020), 553 (554 - 559). 